\title{
Erratum: In Situ Generation of High-Energy Spin-Polarized Electrons in a Beam-Driven Plasma Wakefield Accelerator [Phys. Rev. Lett. 126, 054801 (2021)]
}

Zan Nie®, Fei Li, Felipe Morales, Serguei Patchkovskii, Olga Smirnova, Weiming An, Noa Nambu, Daniel Matteo, Kenneth A. Marsh, Frank Tsung, Warren B. Mori, and Chan Joshi

(Received 29 November 2021; published 20 December 2021)

DOI: 10.1103/PhysRevLett.127.269901

Because of the absence of definition of the laser helicity in the Letter, the labeling of Fig 1(a) should be modified as follows. The original words "Corotating laser field" should be changed to "Counterrotating (Left-handed laser field $\sigma^{-}$)". The original words "Counterrotating laser field" should be changed to "Corotating (Left-handed laser field $\sigma^{-}$)."

The sentence in the abstract, "Using time-dependent Schrödinger equation (TDSE) simulations ... leading to high total spin polarization." should be corrected as: "Using time-dependent Schrödinger equation (TDSE) simulations, we show the propensity rule of spin-dependent ionization of xenon atoms does not flip as a function of photoelectron energy compared with the non-adiabatic tunneling regime, leading to high total spin polarization."

In the sentence (page 2, third paragraph) "Consider first ionization from the $p^{+}$orbital (corotating with the laser field)..." should be changed to "Here, we choose left-handed $\left(\sigma^{-}\right)$circularly polarized pulses both in our analysis and later calculations. Consider first ionization from the $p^{+}$orbital (counterrotating with the laser field)...."

Without loss of generalization, in the sentence (page 3, first paragraph): "the $p^{-}$electrons are more likely to be ionized" should be changed to "counterrotating electrons are more likely to be ionized."

In the sentence (page 3, second paragraph): "From our TDSE simulations, ionization of Xe by the third harmonic $(\lambda=260 \mathrm{~nm})$ of a Ti:sapphire laser is strongly dominated by the removal of a $p^{+}$electron..." should be changed to "From our TDSE simulations, ionization of Xe by a $\lambda=260 \mathrm{~nm}$ left-handed $\left(\sigma^{-}\right)$circularly polarized laser is strongly dominated by the removal of a $p^{+}$electron (counterrotating with the laser field)...."

These changes do not affect either the total spin polarization or the main message of the Letter. 